\title{
THE CLASSIFICATION OF SOME MODULAR FROBENIUS GROUPS
}

\author{
JUANJUAN FAN, NI DU ${ }^{凶}$ and JIWEN ZENG
}

(Received 2 April 2011)

\begin{abstract}
Fix a prime number $p$. Let $G$ be a $p$-modular Frobenius group with kernel $N$ which is the minimal normal subgroup of $G$. We give the complete classification of $G$ when $N$ has three, four or five $p$-regular conjugacy classes. We also determine the structure of $G$ when $N$ has more than five $p$-regular conjugacy classes.
\end{abstract}

2010 Mathematics subject classification: primary 20C20; secondary 20E45.

Keywords and phrases: modular Frobenius groups, minimal normal subgroups, $p$-regular conjugacy classes.

\section{Introduction}

Finite Frobenius groups have played a major role in many areas of group theory, notably in the analysis of doubly transitive groups and finite simple groups. There are many generalizations of finite Frobenius groups. We study the characteristic $p$ analogue of finite Frobenius groups, the so-called $p$-modular Frobenius groups which were introduced by Kuisch and van der Waall in [7] and which they defined as follows.

Definition 1.1. Let $1<N \triangleleft G$. Suppose that $F$ is a splitting field for $F[N]$ with $\operatorname{char}(F)=p>0$. We say that $G$ is a $p$-modular Frobenius group if $G$ satisfies one of the following equivalent conditions:

(i) every nontrivial irreducible $F[N]$-module $V$ has the property that $V^{G}$ is irreducible;

(ii) $C_{G}(x) \leq N$ for every nontrivial $p$-regular element $x$ of $N$.

A normal subgroup $N$, as in Definition 1.1, is called a $p$-modular Frobenius kernel.

It is trivially true that a normal $p$-subgroup of $G$ (having no nontrivial $p$-regular elements) is necessarily a $p$-modular Frobenius kernel. So in this paper, we deal exclusively with $p$-modular Frobenius kernels that are not $p$-groups.

This research was supported by the Fundamental Research Funds for the Central Universities (No. 2010121003).

(C) 2011 Australian Mathematical Publishing Association Inc. 0004-9727/2011 \$16.00 
Let $G$ be a finite group and fix a prime number $p$. Let $F$ be an algebraically closed field of characteristic $p$. It follows that $F$ is a splitting field for every group by [6, Corollary 9.4]. We know that there is a natural one-to-one correspondence between isomorphism classes of $F G$-modules and similarity classes of representations of $F G$. If $\varphi \in \operatorname{IBr}(G)$, then $\varphi$ uniquely determines (and is uniquely determined by) an irreducible $F$-representation $\mathcal{X}$ up to similarity. Therefore we have the following definition which is equivalent to Definition 1.1.

Definition 1.2. A group $G$ is a $p$-modular Frobenius group with kernel $N$ if every nonprincipal irreducible Brauer character of $N$ induces irreducibly to $G$.

As one knows, conjugacy class lengths impose great influences on the structure of finite groups. Alemany et al. determined the structure of $G$ when the set of $p$-regular conjugacy class sizes of $G$ is $\{1, m\}$ for an arbitrary integer $m>1$ in [1]. Let $N$ be a normal subgroup of $G$. The relationship between the number of conjugacy classes of $N$ and that of $G$ was discussed in [3]. Since Brauer characters are only defined on $p$-regular elements, we will focus on the number of $p$-regular conjugacy classes of the $p$-modular Frobenius kernel.

In this paper, we give the classification of $p$-modular Frobenius groups according to the number of $p$-regular conjugacy classes of modular Frobenius kernels which are the minimal normal subgroups.

There is a reason why we consider the case where the $p$-modular Frobenius kernel is a minimal normal subgroup of $G$. Let $G$ be a $p$-modular Frobenius group with kernel $N$ and $H$ be a minimal normal subgroup of $G$. It follows that $H \cap N=1$ or $H \leq N$ by the minimality of $H$. If $H \cap N=1$, then $H \leq C_{G}(x)$ for each element $x$ of $N$. By Definition 1.1, we have $H \leq N$, and hence $H=1$. This is not possible because $H$ is nontrivial. So $H \leq N$. If $H<N$, it follows that $G / H$ is a $p$-modular Frobenius group with kernel $N / H$ by [7, Lemma 2.3]. Suppose that $H_{1} / H$ is a minimal normal subgroup of $G / H$. Then it follows that $H_{1} / H$ is contained in $N / H$ by the above argument. If $H_{1} / H<N / H$, then $G / H_{1}$ is a $p$-modular Frobenius group with kernel $N / H_{1}$ by [7, Lemma 2.3]. We can repeat the process and find $M \triangleleft G$ with $M<N$ such that $G / M$ is a $p$-modular Frobenius group with kernel $N / M$, where $N / M$ is a minimal normal subgroup of $G / M$. So we consider the case where the $p$-modular Frobenius kernel is a minimal normal subgroup of $G$.

Before stating our results, we introduce some notation. Let $G$ be always a finite group. We write $\operatorname{IBr}(G)$ for the set of irreducible Brauer characters of $G$ at the given fixed prime $p$. Let $G^{0}$ be the set of $p$-regular elements of $G$ (that is, the set of elements $g \in G$ such that the order of $g$ is not divisible by $p)$. We write $\operatorname{cl}\left(G^{0}\right)$ for the set of conjugacy classes of $G$ which consist of $p$-regular elements. Also, we shall write $1_{G^{0}}$ for the principal Brauer character of $G$. Denote $Z_{m}$ and $\left(Z_{r}\right)^{n}$ to be a cyclic group of order $m$ and an elementary abelian $r$-group of order $r^{n}$, respectively. Let $\pi(n)$ be the set of all prime divisors of the positive integer $n$. Let $D_{10}$ be the dihedral group of order 10 and $M_{10}$ be the stabilizer of a point in the Mathieu group $M_{11}$ (see [2]). For other notation and terminology, one can refer to $[8,9]$. 


\section{Preliminaries}

In this section, we list some basic properties of modular Frobenius groups and primary lemmas for the proofs of our theorems. The next few results, which are crucial to our investigation, are due to Kuisch and van der Waall [7].

Lemma 2.1. Suppose that $G$ is a p-modular Frobenius group with kernel $N$ and $N$ is a nonabelian simple group. Then $p=2$ and $N \cong A_{6}$.

Lemma 2.2. Suppose that $G$ is a 2-modular Frobenius group with nonsolvable kernel $N$. Then $G / N$ is a 2-group.

Lemma 2.3. Suppose that $G$ is a p-modular Frobenius group, $p$ odd with kernel $N$. Then $N$ is solvable.

For the proofs, see [7, Corollary 3.8, Theorems 3.10 and 4.1].

The next result is general in the modular representation theory of finite groups.

Lemma 2.4. Suppose that $1<N \triangleleft G$ and let $\theta \in \operatorname{IBr}(N)$. Then $\theta^{G} \in \operatorname{IBr}(G)$ if and only if $I_{G}(\theta)=N$, where $I_{G}(\theta)$ is the inertia group of $\theta$ in $G$.

Proof. This is a direct consequence of [9, Problem 8.3] and [5, Theorem 2.2].

The following corollary is immediate by Definition 1.2 and Lemma 2.4.

Corollary 2.5. Suppose that $G$ is a p-modular Frobenius group with kernel $N$. Then $I_{G}(\theta)=N$ for each nonprincipal irreducible Brauer character $\theta$ of $N$.

Corollary 2.6. Suppose that $G$ is a p-modular Frobenius group with kernel N. Let $G$ act on $\operatorname{IBr}(N)$ naturally. Namely, if $\theta \in \operatorname{IBr}(N)$ and $g \in G$, then $\theta^{g}(n)=\theta\left(n^{g^{-1}}\right)$ for each $n \in N^{0}$. Write $\Delta_{\theta}$ for the orbit of $\theta$ for each element $\theta$ in $\operatorname{IBr}(N)$. Then $\left|\Delta_{\theta}\right|=|G: N|$ for each nonprincipal irreducible Brauer character $\theta$ of $N$. Furthermore, $|G: N|$ divides $\left|\mathrm{cl}\left(N^{0}\right)\right|-1$.

Proof. Using Corollary 2.5 and the fact that $\left|G: I_{G}(\theta)\right|$ is the number of distinct $G$-conjugates of $\theta$, it follows that $\left|\Delta_{\theta}\right|=|G: N|$ for each nonprincipal irreducible Brauer character $\theta$ of $N$, and hence $|G: N|$ divides $|\operatorname{IBr}(N)|-1$. The second part follows since $|\operatorname{IBr}(N)|$ is the number of $p$-regular conjugacy classes of $N$.

Corollary 2.7. Suppose that $G$ is a p-modular Frobenius group with kernel $N$. Then each irreducible Brauer character in $\operatorname{IBr}(G) \backslash \operatorname{IBr}(G / N)$ is induced from a nonprincipal irreducible Brauer character of $N$, where

$$
\operatorname{IBr}(G) \backslash \operatorname{IBr}(G / N)=\{\varphi \in \operatorname{IBr}(G): N \not \leq \operatorname{ker} \varphi\} .
$$

Also, if the number of $G$-orbits on $\operatorname{IBr}(N)$ is $k+1$, then $|\operatorname{IBr}(G) \backslash \operatorname{IBr}(G / N)|=k$.

Proof. Let $\varphi \in \operatorname{IBr}(G) \backslash \operatorname{IBr}(G / N)$. By Clifford's theorem, $\varphi_{N}=e \sum_{i=1}^{t} \theta_{i}$, where $\theta_{1}=\theta, \theta_{2}, \ldots, \theta_{t}$ are the distinct conjugates of $\theta$ in $G$. We have $\theta \neq 1_{N^{0}}$. Otherwise, it will contradict the fact that $N=\operatorname{ker} \varphi$. By Definition 1.2, we obtain $\theta^{G}=\varphi$ and the 
first part follows. Applying the first part and Definition 1.2, we have a one-to-one correspondence between the set of $G$-orbits on $\operatorname{IBr}(N) \backslash\left\{1_{N^{0}}\right\}$ and $\operatorname{IBr}(G) \backslash \operatorname{IBr}(G / N)$, where $\operatorname{IBr}(N) \backslash\left\{1_{N^{0}}\right\}$ is the set of nonprincipal irreducible Brauer characters of $N$. Hence the proof of the second part is complete.

Our next objective is to prove that every minimal normal subgroup of a modular Frobenius group is contained in a modular Frobenius kernel.

Lemma 2.8. Suppose that $G$ is a p-modular Frobenius group with kernel $N$. Let $M$ be a nontrivial normal subgroup of $G$. Then $N \cap M>1$. In particular, every minimal normal subgroup of $G$ is contained in $N$. When $p$ is odd, all minimal normal subgroups of $G$ are elementary abelian.

Proof. Assume that $N \cap M=1$. It follows that $M \leq C_{G}(N)$. By Definition 1.1, there exists a nontrivial $p$-regular element $x$ in $N$ such that $C_{G}(x) \leq N$. Hence $M \leq N$. It follows that $M=1$, and this contradicts the fact that $M$ is nontrivial. So $N \cap M>1$. In particular, if $H$ is a minimal normal subgroup of $G$, then $H \leq N$. When $p$ is odd, by Lemma 2.3, $H$ is elementary abelian.

Next, we show that a modular Frobenius kernel is not necessarily a Hall subgroup.

Lemma 2.9. Suppose that $G$ is a p-modular Frobenius group with kernel $N$. Then $(|G / N|,|N|)=p^{a}$ with $a \in \mathbb{N}$.

Proof. Assume that there exists a prime $q \neq p$ such that both $|G / N|$ and $|N|$ are divisible by $q$. Let $Q$ be a Sylow $q$-subgroup of $G$. Since $Q \cap N$ is a nontrivial normal subgroup of $Q$, it follows that $Z(Q) \cap N>1$. Pick a nontrivial element $x$ in $Z(Q) \cap N$. Then $Q \leq C_{G}(x) \leq N$, whence $G / N$ is a $q^{\prime}$-group. But this clearly contradicts the assumption that $|G / N|$ is divisible by $q$, so the proof is complete.

Corollary 2.10. Suppose that $G$ is a p-modular Frobenius group with kernel $N$. Then $G$ is nonnilpotent.

Proof. Assume that $G$ is nilpotent. Let $P$ be the Sylow $p$-subgroup of $G$. Then $P \leq C_{G}(x) \leq N$ for any nontrivial $p$-regular element $x$ of $N$. So by Lemma 2.9, we have $(|G / N|,|N|)=1$. Let $Q$ be any Sylow subgroup of $N$. Then $Q$ is also a Sylow subgroup of $G$. Using Definition 1.1 and the fact that a nilpotent group is a direct product of Sylow subgroups, it follows that $N=G$, a contradiction. We therefore conclude that $G$ is nonnilpotent.

As one knows, a Frobenius group $G$ is a $p$-modular Frobenius group when the Frobenius kernel contains a nontrivial $p$-regular element. By the definition of $p$-modular Frobenius groups, we know that a $p$-modular Frobenius group $G$ with kernel $N$ is a Frobenius group if either $|G|$ or $|N|$ is coprime to $p$. It is not generally true that a $p$-modular Frobenius group is a Frobenius group. A counterexample is $\operatorname{GL}(2,3)$. $\operatorname{GL}(2,3)$ is a 2-modular Frobenius group with kernel $\operatorname{SL}(2,3)$, while $\operatorname{GL}(2,3)$ is not a Frobenius group. So we have a wide class of $p$-modular Frobenius groups by the theory of Frobenius groups. Although $p$-modular Frobenius 
groups need not be Frobenius groups, in the following proposition, we investigate conditions for $p$-modular Frobenius groups to be Frobenius groups.

Proposition 2.11. Suppose that $G$ is a p-modular Frobenius group with kernel $N$ and $(|G / N|,|N|)=1$. If $N$ is a minimal normal subgroup of $G$, then $G$ is a Frobenius group with elementary abelian kernel $N$.

Proof. Assume that $p$ divides $|N|$. If $p$ is odd, then $N$ is solvable by Lemma 2.3. It follows that $N$ is an elementary abelian $r$-group with $r \neq p$ by the minimality of $N$. This contradicts the assumption. Now we have $p=2$. When $N$ is solvable, we can obtain the same contradiction as above. If $N$ is nonsolvable, then $G / N$ is a 2-group by Lemma 2.2, and hence both $|G / N|$ and $|N|$ are divisible by 2 . This is in contradiction to $(|G / N|,|N|)=1$. So $p$ does not divide $|N|$. It follows that $G$ is a Frobenius group with kernel $N$, and we conclude that $N$ is nilpotent. The desired conclusion follows.

A well-known result about Frobenius group is about the center subgroup. We now describe the relationship between the center subgroup of a $p$-modular Frobenius group and the $p$-modular Frobenius kernel.

Lemma 2.12. Suppose that $G$ is a p-modular Frobenius group with kernel $N$. Then $Z(G)<N$. Furthermore, $Z(G)$ is a p-group.

Proof. Let $x$ be a nontrivial $p$-regular element of $N$. Then $C_{G}(x) \leq N$. It follows that $Z(G) \leq N$. If $Z(G)=N$, then $G=C_{G}(x) \leq N$. This is impossible. So $Z(G)<N$. Suppose that there exists a nontrivial $p$-regular element $y$ in $Z(G)$. Then $G=C_{G}(y) \leq N$, but $N<G$, a contradiction. Therefore $Z(G)$ is a $p$-group.

\section{Main results}

Let $(*)$ be the hypothesis:

(*) $\quad G$ is a p-modular Frobenius group with kernel $N$ and $N$ is a minimal normal subgroup of $G$.

In this section, we will determine the structure of $G$ according to the number of $p$-regular conjugacy classes of $N$ under the hypothesis $(*)$. We begin with a general result for all modular Frobenius groups.

Proposition 3.1. Suppose that $G$ is a p-modular Frobenius group with kernel $N$. Then the number of p-regular conjugacy classes of $N$ is at least three.

Proof. Since $N$ is not a $p$-group, by Cauchy's theorem, there exists a prime divisor $r$ of $|N|$ such that $N$ contains an element of order $r$, where $r \neq p$. It follows that $\left|\operatorname{cl}\left(N^{0}\right)\right| \geq 2$. Assume $\left|\operatorname{cl}\left(N^{0}\right)\right|=2$. Then we can $\operatorname{set} \operatorname{IBr}(N)=\left\{1_{N^{0}}, \theta\right\}$. Let $G$ act on $\operatorname{IBr}(N)$ naturally as Corollary 2.6. Then $\theta$ is $G$-invariant. Using Corollary 2.5, we have a contradiction. 
Now we give the complete characterization when $N$ contains three, four or five $p$-regular conjugacy classes.

Theorem 3.2. Assume (*). If $\left|\operatorname{cl}\left(N^{0}\right)\right|=3$, then $G \cong S_{3}$.

Proof. We can set $\operatorname{IBr}(N)=\left\{1_{N^{0}}, \theta_{1}, \theta_{2}\right\}$. Let $G$ act on $\operatorname{IBr}(N)$ naturally as Corollary 2.6. Then $\theta_{1}$ is $G$-conjugate to $\theta_{2}$ by Corollary 2.5. This implies that $|G: N|=2$ by Corollary 2.6 and the number of $G$-orbits on $\operatorname{IBr}(N)$ is two. Now we distinguish two cases: $p$ is even and $p$ is odd.

If $p$ is odd, then $N$ is solvable by Lemma 2.3. By the minimality of $N$, we have that $N$ is an elementary abelian $r$-group with $r \neq p$. It follows that $p$ does not divide the order of $N$, and so $G$ is a Frobenius group with kernel $N$. Notice that $N$ is an abelian $p^{\prime}$-group and $\left|\operatorname{cl}\left(N^{0}\right)\right|=3$. We can obtain $|N|=\left|\operatorname{cl}\left(N^{0}\right)\right|=3$. Hence $N \cong Z_{3}$. Since $G$ is a Frobenius group with kernel $N$ and $|G: N|=2$, it follows that $G \cong Z_{3} \rtimes Z_{2}$.

If $p=2$, then $\operatorname{IBr}(G / N)=\left\{1_{G^{0}}\right\}$ follows from $|G: N|=2$. Since the number of $G$-orbits on $\operatorname{IBr}(N)$ is two, it follows from Corollary 2.7 that $|\operatorname{IBr}(G) \backslash \operatorname{IBr}(G / N)|=1$. Notice that $\operatorname{IBr}(G / N)=\left\{1_{G^{0}}\right\}$. We see that $G$ has exactly two irreducible Brauer characters, and hence $G$ has exactly two 2-regular conjugacy classes. As $|G: N|=2$, by Cauchy's theorem, the order of $G$ has just two different prime divisors, one of which is 2. Applying the famous solvability criterion of Burnside, we conclude that $G$ is solvable, and hence $N$ is solvable. The remainder of the proof is similar to the second paragraph, and hence we also obtain $G \cong S_{3}$.

Theorem 3.3. Assume (*). If $\left|\operatorname{cl}\left(N^{0}\right)\right|=4$, then $G \cong A_{4}$.

Proof. Let $G$ act on $\operatorname{IBr}(N)$ as Corollary 2.6. Since $|\operatorname{IBr}(N)|=\left|\operatorname{cl}\left(N^{0}\right)\right|=4$, we have $|G: N|=3$ by Corollary 2.6. Assume $p=2$. If $N$ is nonsolvable, then $G / N$ is a 2-group by Lemma 2.2. This is in contradiction to $|G: N|=3$. If $N$ is solvable, then $N$ is an elementary abelian $r$-group with $r \neq 2$ by the minimality of $N$. It follows that $\operatorname{cl}\left(N^{0}\right)=\operatorname{cl}(N)$ and $|N|=4$. But $N$ is an $r$-group with $r \neq 2$, a contradiction. Hence $p$ is odd, whence $N$ is solvable by Lemma 2.3. The same argument shows that $|N|=4$. So $N$ is isomorphic to the Klein 4-group $K_{4}$ and $G$ is a Frobenius group with kernel $N$. Since $|G: N|=3$, we have $G \cong K_{4} \rtimes Z_{3}$, so the proof is complete.

Theorem 3.4. Assume (*). If $\left|\operatorname{cl}\left(N^{0}\right)\right|=5$, then either $G \cong D_{10}$, or $G \cong M_{10}$, or $G \cong$ $Z_{5} \rtimes Z_{4}$.

Proof. Notice that $\left|\operatorname{cl}\left(N^{0}\right)\right|=5$ and $N$ is the minimal normal subgroup of $G$. Since $N$ is a direct product of isomorphic simple groups, we can conclude that $N$ is a simple group. Let $G$ act on $\operatorname{IBr}(N)$ as Corollary 2.6. As $|\operatorname{IBr}(N)|=\left|\operatorname{cl}\left(N^{0}\right)\right|=5$, we can see that $|G: N|=2$ or $|G: N|=4$ by Corollary 2.6. So there are two cases to distinguish.

(i) The case when $|G: N|=2$.

When $p$ is odd, $N$ is solvable by Lemma 2.3. Since $N$ is simple, it follows that $N$ is a cyclic group of prime order $r$ which is different from $p$. So $G$ is a Frobenius group with kernel $N$. Notice that $\left|\operatorname{cl}\left(N^{0}\right)\right|=5$. We have $|N|=\left|\operatorname{cl}\left(N^{0}\right)\right|=5$, and hence $N \cong Z_{5}$. As $G$ is a Frobenius group with kernel $N$ and $|G: N|=2$, 
we have $G \cong D_{10}$, where $p \neq 5$. Now let $p=2$. When $N$ is solvable, we repeat the above process and get $G \cong D_{10}$. Suppose that $N$ is nonsolvable. Since $N$ is simple, it follows that $N \cong A_{6}$ by Lemma 2.1. Notice that $|G: N|=2$. Using the small groups library in GAP (see [4]), we can get $G \cong M_{10}$.

(ii) The case when $|G: N|=4$.

When $p$ is odd, $N$ is solvable by Lemma 2.3. The above argument shows that $N \cong Z_{5}$ and $G$ is a Frobenius group with kernel $N$. Now, $G / N$ is isomorphic to an abelian Frobenius complement since $|G: N|=4$. By the structure theorem on Frobenius groups (see [10, Theorem 10.5.6]), the Frobenius complement of $G$ has to be cyclic. So $G \cong Z_{5} \rtimes Z_{4}$ with $p \neq 5$. When $p$ is even, $\operatorname{IBr}(G / N)=$ $\left\{1_{G^{0}}\right\}$ follows from $|G: N|=4$. Notice that $|\operatorname{IBr}(N)|=5$ and $|G: N|=4$. From Corollary 2.6 it follows that the number of $G$-orbits on $\operatorname{IBr}(N)$ is two. Consequently $|\operatorname{IBr}(G)|=2$ by Corollary 2.7. As $G$ has exactly two $p$-regular conjugacy classes and $|G: N|=4$, by Cauchy's theorem, we have that $|G|$ has just two prime divisors, one of which is 2. Applying Burnside's theorem, we conclude that $G$ is solvable, and hence $N$ is solvable. Similarly, we obtain that $G$ is a Frobenius group which is isomorphic to $Z_{5} \rtimes Z_{4}$.

In the following, we will consider the case where $\left|\operatorname{cl}\left(N^{0}\right)\right| \geq 6$.

Theorem 3.5. Assume (*). If $\left|\operatorname{cl}\left(N^{0}\right)\right|$ is even, then $\left|\operatorname{cl}\left(N^{0}\right)\right|=2^{a}$. Furthermore, $G$ is a Frobenius group with kernel $\left(Z_{2}\right)^{a}$.

Proof. Assume that $\left|\operatorname{cl}\left(N^{0}\right)\right|=2^{a} b$, where 2 does not divide $b$. If $N$ is solvable, then $N$ is an elementary abelian $r$-group with $r \neq p$ by the minimality of $N$. So $\left|\operatorname{cl}\left(N^{0}\right)\right|=|\operatorname{cl}(N)|=|N|=2^{a} b$. But this contradicts the fact that $N$ is an $r$-group. Hence $N$ is nonsolvable. By Lemma 2.3, $p=2$. Then $G / N$ is a 2-group by Lemma 2.2. By Corollary 2.6, we have a contradiction since $|G / N|$ divides $\left(2^{a} b-1\right)$ which is odd.

Now $\left|\operatorname{cl}\left(N^{0}\right)\right|=2^{a}$. We claim that $N$ is solvable. Otherwise, $p=2$ by Lemma 2.3. It follows that $G / N$ is a 2-group by Lemma 2.2. By Corollary 2.6, we have a contradiction since $|G: N|$ divides $\left(2^{a}-1\right)$ which is odd, as claimed. By the minimality of $N$, we obtain $N$ is an elementary abelian $r$-group with $r \neq p$. So $\left|\operatorname{cl}\left(N^{0}\right)\right|=|\operatorname{cl}(N)|=$ $|N|=2^{a}$ and $G$ is a Frobenius group with kernel $N$. The result follows.

Theorem 3.6. Assume (*). If $\left|\operatorname{cl}\left(N^{0}\right)\right|$ is odd, then either $G$ is a Frobenius group with elementary abelian kernel $N$, or $N$ is a direct product of isomorphic PSL $\left(2,3^{2^{k}}\right)$ for some positive integer $k$.

Proof. If $N$ is solvable, then $N$ is an elementary abelian $r$-group with $r \neq p$ by the minimality of $N$, whence $\pi(|N|)=1$. It follows that $G$ is a Frobenius group with kernel $N$. If $N$ is nonsolvable, then $p=2$ by Lemma 2.3. We claim that $N$ is nonsimple. Otherwise, $N \cong A_{6}$ by Lemma 2.1. Notice that $\left|\operatorname{cl}\left(N^{0}\right)\right| \geq 6$. This contradicts the fact that the number of 2-regular conjugacy classes of $A_{6}$ is five, as claimed. As $N$ is a direct product of isomorphic simple groups, the result follows by [7, Theorem 3.7]. 


\title{
Acknowledgement
}

We would like to thank the referee for his or her helpful comments.

\section{References}

[1] E. Alemany, A. Beltrán and M. J. Felipe, 'Finite groups with two $p$-regular conjugacy class lengths II', Bull. Aust. Math. Soc. 79 (2009), 419-425.

[2] J. D. Dixon and B. Mortimer, Permutation Groups (Springer, New York, 1996).

[3] P. X. Gallagher, 'The number of conjugacy classes in a finite group', Math. Z. 118 (1970), $175-179$.

[4] The GAP Group, 'GAP - Groups, Algorithms, and Programming', Version 4.4, 2006, http://www.gap-system.org.

[5] I. M. Isaacs, 'Lifting Brauer characters of $p$-solvable groups', Pacific J. Math. 53 (1974), 171-188.

[6] I. M. Isaacs, Character Theory of Finite Groups (Academic Press, New York, 1976).

[7] E. B. Kuisch and R. W. van der Waall, 'Modular Frobenius groups', Manuscripta Math. 90 (1996), 403-427.

[8] H. Kurzweil and B. Stellmacher, The Theory of Finite Groups: An Introduction (Springer, New York, 2004).

[9] G. Navarro, Characters and Blocks of Finite Groups (Cambridge University Press, Cambridge, 1978).

[10] D. J. S. Robinson, A Course in the Theory of Groups (Springer, New York, Berlin, 2003).

\author{
JUANJUAN FAN, School of Mathematical Sciences, Xiamen University, \\ Xiamen, PR China \\ e-mail: maygaxm@hotmail.com
}

NI DU, School of Mathematical Sciences, Xiamen University, Xiamen, PR China e-mail: duni@xmu.edu.cn

JIWEN ZENG, School of Mathematical Sciences, Xiamen University, Xiamen, PR China

e-mail: jwzeng@xmu.edu.cn 